\title{
Sigmoid volvulus in young patients: Ac new twist on an old diagnosis
}

\author{
Moaziz Sarfaraz ${ }^{1, *}$, Syeda Rana Hasan ${ }^{2}$, Shahid Lateef ${ }^{3}$ \\ ${ }^{1}$ Emergency Department, Fujairah Hospital, Fujairah, United Arab Emirate; \\ ${ }^{2}$ Department of Preventive Medicine, Fujairah, United Arab Emirate; \\ ${ }^{3}$ Department of Surgery, Kalba Hospital, Kalba, Sharjah, United Arab Emirate.
}

\begin{abstract}
Summary According to the literature, sigmoid volvulus typically develops in patients of an older age with co-morbidities such as a psychiatric illness or a bed-bound chronic illness. Recent reports suggest that it should also be considered in young individuals without any preceding medical history. Abdominal roentgenography is a cheap and effective diagnostic modality that can avoid a delay in diagnosis. The treatment of colonic volvulus remains controversial and relies upon the procedure selected and the most appropriate therapeutic approach in terms of the clinical status of the patient, the location of the problem, the suspected existence or identification of peritonitis, bowel viability, and the expertise of the surgical team. Presented here are four cases of young male patients with sigmoid volvulus. All of the patients were diagnosed radiologically prior to surgical intervention. Two of the patients initially underwent an endoscopic procedure that succeeded in one and that failed in the other. Three of the patients underwent a laparotomy.
\end{abstract}

Keywords: Ischemia, laparotomy, obstipation, roentgenogram, sigmoid volvulus, strangulation, torsion

\section{Introduction}

Large bowel volvulus is an uncommon cause of bowel obstruction in the industrialized world (1). However, it is four times more common in the Middle East than in Western countries (2). It constitutes the third leading cause of large bowel obstruction in Western countries after carcinoma and diverticulitis (3-5). India, Iran, and Russia have one of the higher incidences of sigmoid volvulus (6). Sigmoid volvulus occurs when the sigmoid colon twists axially around a narrow base of mesentery, leading to a close loop of bowel that is at risk of strangulation, ischemia, gangrene, and perforation $(7,8)$.

Sigmoid volvulus is the most common cause of strangulation of the colon (4). It constitutes less than $10 \%$ of bowel obstructions in industrialized countries, compared with a rate as high as $25 \%$ in the developing regions of the world (9). Sigmoid volvulus, which often

Released online in J-STAGE as advance publication August 7, 2017.

*Address correspondence to:

Dr. Moaziz Sarfaraz, Emergency Department, Fujairah

Hospital, Fujairah, U.A.E.

E-mail:m4moaziz@yahoo.com develops during the $7^{\text {th }}$ and $8^{\text {th }}$ decades of life, has been reported in young people as well (10) Oren et al. (11) found that the mean age of its incidence was 58 years. Liang et al. (12) corroborated that finding, and Liang et al. noted the highest prevalence of sigmoid volvulus in the $7^{\text {th }}$ decade of life. Slidell et al. (13) reported three teenagers ages 17-19 with sigmoid volvulus. A 19-year-old male with the same signs and symptoms was encountered by Salinas et al. (7). Reported here are four male patients with a mean age of 27.5 years who had abdominal pain and obstipation and who were diagnosed with acute sigmoid volvulus.

\section{Case Report}

\subsection{Case 1}

A 25-year-old Indian laborer presented to the Emergency Department with abdominal pain and absolute constipation for three days. The man occasionally vomited as well. The pain was colicky in nature. The man had no past medical history. He was afebrile with a distended abdomen and mild generalized tenderness. Bowel sounds were exaggerated. A rectal digital examination revealed an empty rectum. Abdominal 


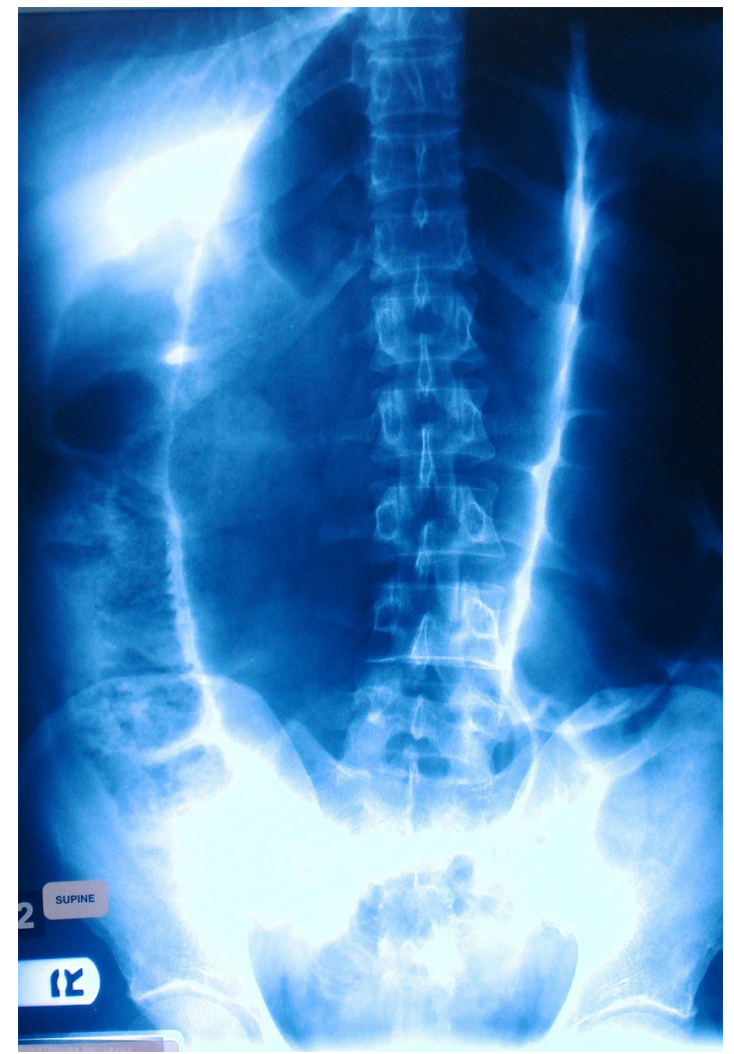

Figure 1. Classic coffee-bean sign on plain abdominal radiograph in Case No. 1.

roentgenography revealed a classic sign of sigmoid volvulus (coffee-bean sign) (3) (Figure 1). The man's WBC count was $18,000 / \mathrm{mm}^{3}\left(4-11 \mathrm{~mm}^{3}\right)$ with a neutrophil percentage $80 \%$. Serum electrolytes were: sodium $130 \mathrm{mmol} / \mathrm{L}(134-145 \mathrm{mmol} / \mathrm{L})$, potassium 3.0 $\mathrm{mmol} / \mathrm{L}(3-5 \mathrm{mmol} / \mathrm{L})$, urea $5.5 \mathrm{mmol} / \mathrm{L}(2.9-9.3 \mathrm{mmol} /$ $\mathrm{L})$, and creatinine $110 \mathrm{micromol} / \mathrm{L}$ (80-115 micrmil/ L). Detorsion was attempted endoscopically but unsuccessful. A laparotomy was performed, revealing sigmoid volvulus.

\subsection{Case 2}

A 25-year-old male of Arab origin presented to the Emergency Department with colicky abdominal pain. The man had no past medical history. He vomited Twice during the last couple of hours he was seen. The man had not moved his bowels for one week. His abdomen was moderately distended and globally tender. Bowel sounds were audible. A digital rectal examination revealed that the rectum was empty. The man had previously experienced three episodes of abdominal pain. Those episodes were of lesser severity and responded to analgesics. Analgesics failed to relieve the man's pain, so he sought medical attention.

\subsection{Case 3}

A 25-year-old male from the Indian sub-continent presented to the Emergency Department after two days of central abdominal pain along with nausea and obstipation. Two years ago, the man suffered the same problem, but it was fortunately relieved by oral analgesics. When he was seen by the Emergency Department, he was afebrile. An abdominal examination revealed a distension with tympanitic percussion. Bowel sounds were present. Plain abdominal radiography revealed the coffee-bean sign indicative of sigmoid volvulus (3). Colonoscopy was attempted but was unsuccessful. A laparotomy was ultimately performed.

\subsection{Case 4}

A 35-year-old male from the Indian sub-continent was seen by the Emergency Department after multiple episodes of vomiting (yellow-colored) along with generalized abdominal pain lasting three days. The man had no past medical history. Clinically, he was sickly looking. His WBC count was $20,000 / \mathrm{mm}^{3}$ with a neutrophil percentage of $84 \%$. Serum electrolytes were: sodium $128 \mathrm{mmol} / \mathrm{L}$, potassium $2.8 \mathrm{mmol} / \mathrm{L}$, urea $10.5 \mathrm{mmol} / \mathrm{L}$, and creatinine $115 \mathrm{micromol} / \mathrm{L}$. His abdomen was distended with tympanitic percussion. Bowel sounds were hyperactive. Initial plain abdominal radiography suggested sigmoid volvulus. A plain abdominal CT scan revealed the same features (Figure 2 and Figure 3). Colonoscopy revealed an obstruction $45 \mathrm{~cm}$ from the anal verge.

\section{Discussion}

Large bowel volvulus is more prevalent in the developing world, where it constitutes nearly $50 \%$ of all bowel obstructions as compared to only $5 \%$ in the developed world. In the Middle East alone, sigmoid volvulus is four times as common as it is in the Western world $(1,2)$. There are a few contributing factors that are assumed to lead to large bowel volvulus in the Gulf region. The first is the dietary habits of the local population. People consume more junk food, less fiber (vegetables and fruits), and even less water. In addition, hot and humid weather leads to greater water loss from the body. Above all, a sedentary lifestyle is a leading factor for the development of sigmoid volvulus. Sigmoid Volvulus is more common in older patients, and especially those with a psychiatric co-morbidity $(14,15)$. Sigmoid volvulus is also seen in patients suffering from Parkinson's disease, Alzheimer's disease, pseudobulbar palsy, and chronic schizophrenia (3). Oren et al. (11) reported a large series of 827 patients with a mean age of 57.9 years. Tiah et al. (16) studied 28 patients with an average age of 74 years and Liang et al. (12) studied 14 patients with an average age of $68.4 \pm 12.2$ years. In a study of 32 patients by Heis et al. (17), only two were under the age of 30; out of 30 cases of acute sigmoid volvulus, Sule et al. (18) found that 4 patients 


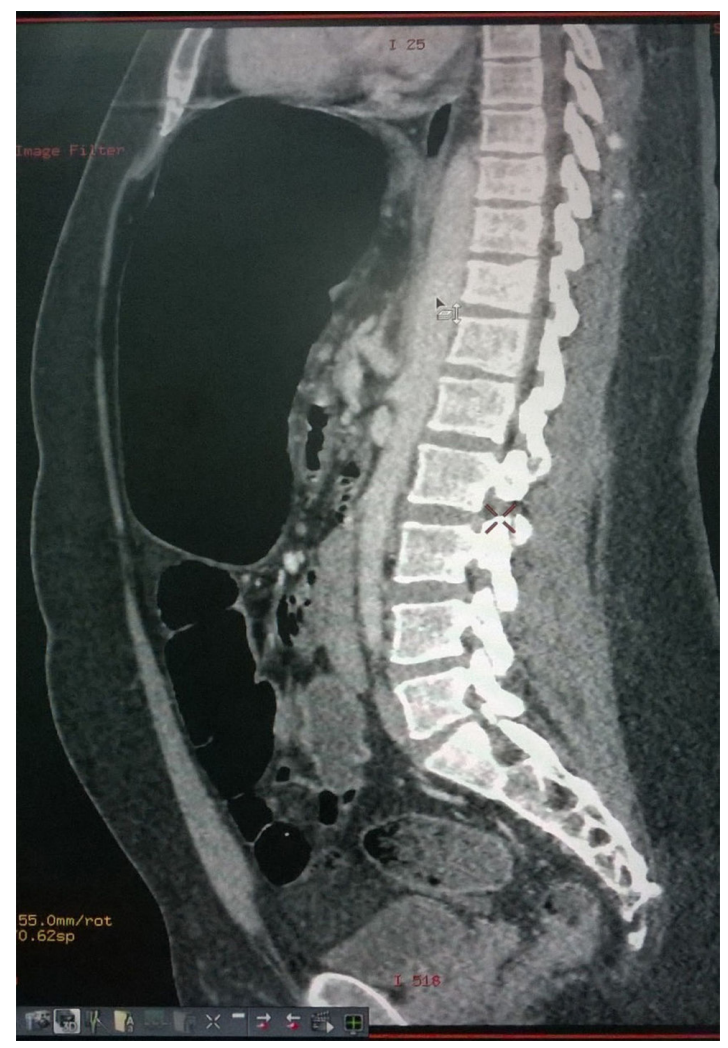

Figure 2. CT image of sigmoid volvulus in Case No. 4.

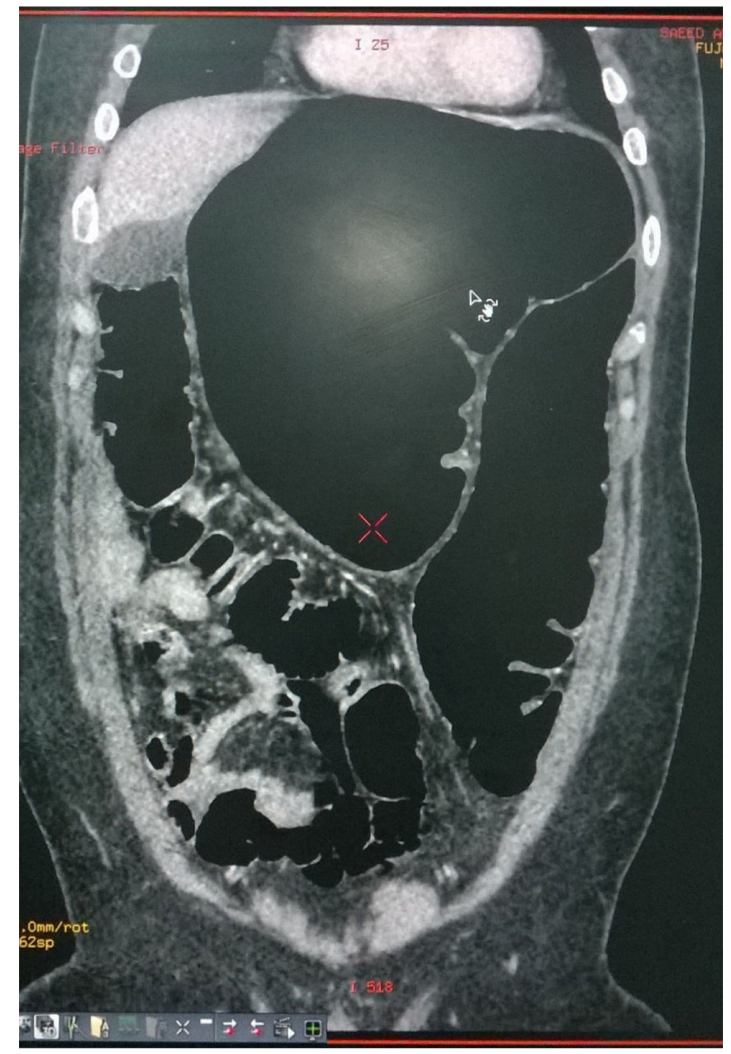

Figure 3. CT image of sigmoid volvulus in Case No. 4.

Table 1. Comparison of the number, gender, and mean age of patients in different studies

\begin{tabular}{|c|c|c|c|c|c|}
\hline \multirow{2}{*}{ No. } & \multirow{2}{*}{ Lead author of the study } & \multicolumn{3}{|c|}{ Number of patients } & \multirow{2}{*}{ Mean age (yrs) } \\
\hline & & Male & Female & Total & \\
\hline 1 & Sarfaraz M (2017) & 4 & 0 & 4 & 27.5 \\
\hline 2 & Chalya PL (2015) & 122 & 24 & 146 & 48 \\
\hline 3 & Salinas NL (2007) & 1 & 0 & 1 & 19 \\
\hline 4 & Oren D (2007) & 688 & 139 & 827 & 57.9 \\
\hline 5 & Tiah L (2006) & 16 & 12 & 28 & 74 \\
\hline 6 & Liang JT (2006) & 10 & 4 & 14 & $68.4 \pm 12.2$ \\
\hline 7 & Agaoglu N (2005) & 21 & 11 & 32 & $73.5 \pm 8.38$ \\
\hline 8 & Slidell M (2004) & 3 & 0 & 3 & 18 \\
\hline 9 & Raveenthiran V (2004) & 42 & 39 & 81 & 50 \\
\hline 10 & Turan M (2003) & 61 & 20 & 81 & 65 \\
\hline
\end{tabular}

were between the ages of 20-30 years. Slidell et al. (13) reported three teenagers with sigmoid volvulus. Salinas et al. (7) encountered a 19-year-old male with the same condition. In a study of 28 patients with acute sigmoid volvulus by Lou et al. (19), 19 were male and 9 were female with a mean age of $63.1 \pm 22.9$ years. All of the current patients were male. Three were 25 years old and one was 35 years old, representing a mean age of 27.5 years. All of the current patients were otherwise healthy with no noticeable medical illness (Table 1). Three of the current patients $(75 \%)$ presented after 2-3 days while one was brought to the Emergency Department within a few hours of the onset of abdominal pain and vomiting.

Classically, sigmoid volvulus can be divided into two subtypes on clinical grounds, as described by
Hinshaw and Carter (20). Acute fulminating volvulus, caused by complete obstruction, clinically presents as sudden onset of central abdominal pain accompanied by emesis and constipation. Gangrene and perforation are common early complications of this type of volvulus. With subacute progressive volvulus, patients have only a partial obstruction and more insidious onset. Older patients frequently have the subacute form (21). The patients encountered by Heis et al. (17) presented with abdominal pain $(96 \%)$, distension $(84 \%)$, vomiting (72\%), and constipation (63\%). Kuzu et al. (22) noted abdominal distension in $89 \%$ of patients and vomiting in $64 \%$. All of the current patients had abdominal pain, $75 \%$ vomited, and one only had nausea. Fifty percent of the current patients had constipation and $50 \%$ had 
obstipation. All were afebrile.

Plain abdominal X-rays are a rapid and useful diagnostic tool $(4,23)$. The classic coffee-bean sign on plain abdominal radiographs was found in $68.7 \%$ of patients encountered by Heis et al. (17) and in $68.5 \%$ of patients encountered by Khanna et al. (24). Javors et al. (25) found that sigmoid volvulus was diagnosed with X-rays in $87 \%$ of patients while Welch and Anderson (14) found that it was similarly diagnosed in $83 \%$ of patients. Tiah et al. (16) reported that sigmoid volvulus was diagnosed with plain abdominal radiography alone in $34.6 \%$ of cases. In suspected cases, a barium enema is recommended both as a diagnostic and therapeutic tool (26). CT provides the additional advantage of excluding other causes of intestinal obstruction in uncertain cases and also facilitating diagnosis of complications, such as a perforation (27). Early diagnosis can prevent ischemia or perforation (25) particularly in younger patients, in whom the chances of a misdiagnosis or a delayed diagnosis are higher (13).

A redundant segment of colon with a short mesentry and close proximity to the point of fixation to the segment are predisposing factors for the development of volvulus (2). Torsion of the sigmoid colon occurs along its mesenteric axis and axial torsion occurs around the axis of the bowel, leading to volvulus (28). The ileosigmoid knot is a rare but serious abdominal emergency in which the ileum and sigmoid entangle each other to form a knot; this can lead to vascular compromise and gangrene of both the ileum and sigmoid colon (21). Venous congestion compromises the colonic blood supply, which occasionally alleviates venous infarction and gangrene. Although less common, involvement of the arterial blood supply can expedite colonic ischemia.

The treatment of sigmoid volvulus remains controversial. It depends on the procedure selected in light of the clinical status of the patient, the location Of the problem, the suspected existence or identification of peritonitis, bowel viability and, last but not least, the expertise of the surgical team (21). Surgeons generally have two surgical options. First, a single-stage procedure in which, initially, endoscopic derotation is followed by semi-elective sigmoidectomy and primary anastomosis (29). The second option is available when decompression fails and there are signs of colonic gangrene. This surgery is in two stages. In the first, a sigmoid resection and Hartman's procedure or a doublebarreled colostomy is performed in order to avoid a high rate mortality with primary anastomosis in that situation. In the second stage 6-8 weeks later, Hartman's procedure is reversed or the colostomy is closed $(30,31)$.

Presented here are unconventional cases of four young patients with a median age of 27.5 years. All of those patients had cramping abdominal pain, usually of several days' duration. One of the patients experienced pain a few hours prior. Common symptoms among the patients were absolute constipation for a couple of days with occasional vomiting. The diagnostic tool Used was simple abdominal radiography. Three patients underwent Hartman's procedure and one was managed with sigmoidoscopic decompression and semielective single-stage sigmoid resection with primary anastomosis.

\section{Conclusion}

Acute sigmoid volvulus is a common differential diagnosis in older patients who are bed-bound or who have a psychiatric co-morbidity. This condition should also be included in the differential diagnosis of young patients with colicky abdominal pain and absolute constipation. The chances of a misdiagnosis or a delayed diagnosis are greater when the symptoms are mild and recurrent. Plain abdominal radiography is a simple, inexpensive, and widely available diagnostic tool that should be used to screen for this rare but serious condition.

\section{References}

1. Lau K, Miller B, Schache D, Cohen J. A study of largebowel volvulus in urban Australia. Can J Surg. 2006; 49:203-207.

2. Turan M, Sen M, Karadayi K, Koyuncu A, Topcu O, Yildirir C, Duman M. Our sigmoid colon volvulus experience and benefits of colonoscope in detortion process. Rev Esp Enferm Dig. 2004; 96:32-35.

3. Feldman D. The coffee bean sign. Radiology. 2000; 216:178-179.

4. Safioleas M, Chatziconstantinou C, Felekouras E, Stamatakos M, Papaconstantinou I, Smirnis A, Safioleas $\mathrm{P}$, Kostakis A. Clinical consideration and therapeutic strategy for sigmoid volvulus in elderly: A study of 33 cases. World J Gastroenterol. 2007; 13:921-924.

5. Lal SK, Morgenstern R, Vinjirayer EP, Martin A. Sigmoid volvulus an update. Gastrointest Endosc Clin N Am. 2006; 16:175-187.

6. Ballantyne GH, Brander MD, Beart RW, Ilstrup DM. Volvulus of colon: Incidence and mortality. Ann Surg. $185 ; 202: 83-92$.

7. Salinas NL, Carr S, Han D, Mahmoud NN. A surprising twist to an old problem: Sigmoid volvulus in a 19-yearold man. Am Surg. 2007; 73:284-286.

8. Gallagher P, Clark K. The ethics of surgery in the elderly demented patient with bowel obstruction. J Med Ethics. 2002; 28:105-108.

9. Madiba TE, Thomson SR. The management of sigmoid volvulus. J R Coll Surg Edinb. 2000; 45:74-80.

10. Sturzaker HG, Lawrie RS, Joiner CL. Recurrent sigmoid volvulus in young people: A missed diagnosis. British Medical Journal. 1975; 4:338-339.

11. Oren D, Atamanalp SS, Aydinli B, Yildirgan IM, Basoglu M, Polat YK, Onbas O. An algorithm of the management of sigmoid colon volvulus and the safety of primary resection: Experience with 827 cases. Dis Colon Rectum. 2007; 50:489-497.

12. Liang JT, Lai HS, Lee PH. Elective laparoscopically assisted sigmoidectomy for the sigmoid volvulus. Surg 
Endosc. 2006; 20:1772-1773.

13. Slidell M, Shah S, Feller E. Sigmoid volvulus in three college age teenagers. J Clin Gastroenterol. 2004; 38:910-911.

14. Welch GH, Anderson JR. Acute volvulus of the sigmoid colon. World J Surg. 1987; 11:258-262.

15. Conolly S, Brannigan AE, Haffemen E, Hayland JMP. Sigmoid volvulus: A 10-year-audit. Ir J Med Sci. 2002; 171:216-217.

16. Tiah L, Goh S H. Sigmoid volvulus: Diagnostic twists and turns. Eur J Emerg Med. 2006; 13:84-87.

17. Heis H, Bani-Hani K, Rabadi D, Elheis M, Bani-Hani B, Mazahreh T, Bataineh Z, Al-Zoubi N, Obeidallah M. Sigmoid volvulus in the Middle East. World J Surg. 2008; 32:459-464.

18. Sule AZ, Iya D, Obekpa PO. Ogbonna B, Momoh JT, Ugwu BT. One stage procedure in the management of acute sigmoid volvulus. J R Coll Edinb. 1999; 44:164166.

19. Lou Z, Yu ED, Zhang W, Meng RG, Hao LQ, Fu CG. Appropriate treatment of acute sigmoid volvulus in the emergency setting. World J Gastroenterol. 2013; 19:49794983.

20. Hinshaw DB, Carter R. Surgical management of acute volvulus of the sigmoid colon: A study of 55 cases. Ann Surg. 1957; 146:52-60.

21. Chalya PL, Mabula JB. Sigmoid volvulus and ileosigmoid knotting: A five-year experience at a tertiary care hospital in Tanzania. World J Emerg Surg. 2015; 10:10.

22. Kuzu MA, Aşlar AK, Soran A, Polat A, Topcu O, Hengirmen $\mathrm{S}$. Emergent resection for acute sigmoid volvulus: Results of 106 consecutive cases. Dis Colon Rectum. 2002; 45:1085-1090.

23. De U. Sigmoid volvulus in rural Bengal. Trop Doct. 2002; 32:80-82.

24. Khanna AK, Kumar P, Khanna R. Sigmoid volvulus: Study from a north Indian hospital. Dis Colon Rectum. 1999; 42:1081-1084.

25. Javors BR, Baker SR, Miller JA. The northern exposure sign: A newly described finding in sigmoid volvulus. AJR. 1999; 173:571-574.

26. Raveenthiran V. Emptiness of the left iliac fossa: A new clinical sign of sigmoid volvulus. Postgrad Med J. 2000; 76:638-641.

27. Wai CT, Lau G, Khor CJL. Clinics in diagnostic imaging (105): Sigmoid volvulus causing intestinal obstruction, with successful endoscopic decompression. Singapore Med J. 2005; 46:483-487.

28. Meyers MA, Ghahremani GG, Govoni AF. Ischemic colitis associated with sigmoid volvulus: New observations. Am J Roentgenol. 1977; 128:591-595.

29. Sule AZ, Ajibade A. Adult large bowel obstruction: A review of clinical experience. Ann Afr Med. 2011; 10:45-50.

30. Nuhu A, Jah A. Acute sigmoid volvulus in a West African population. Ann Afr Med. 2010; 9:86-90.

31. Weingrow D, McCague A, Shah R, Lalezarzadeh F. Delayed presentation of sigmoid volvulus in a young woman. West J Emerg Med. 2012; 13:100-102.

(Received June 3, 2017; Revised July 14, 2017; Accepted July 20, 2017) 